\title{
Nanjing University Institute for the Study of Asian Classics in Chinese
}

\author{
ZHANG Bowei*
}

Institute for the Study of Asian Classics in Chinese, Nanjing University was founded in 2000. In the past decade, under the leadership of Director Prof. Zhang Bowei, it shows a strong momentum of development. Currently, there are four full-time research faculties, several part-time researchers and consultants. When established, the Institute takes study on and collation of Sinographic Texts as its main objective. The so-called 域外漢籍 “Asian Classics in Chinese" or "Sinographic Texts", or "The History of Publications and the Interchange of Literature in the Chinese Cultural Sphere" mainly includes three types: the foreign scholars' historic books written in Chinese language, the

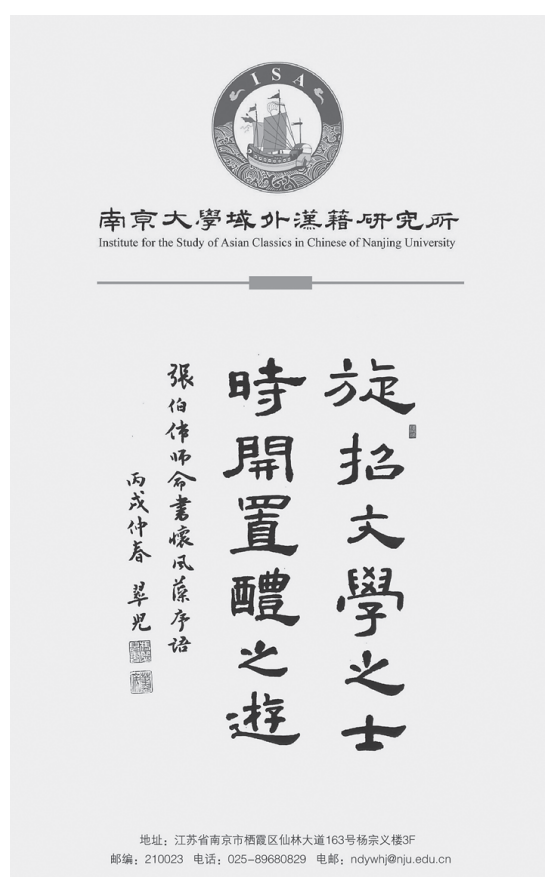
Chinese classics' foreign publications or transcripts, and the lost ancient Chinese classics found overseas. However, the research work of the institute is not only limited to the study of classics, but also the study of cultural exchange and cultural interaction carried and enriched by the classics.

In the past ten years after the establishment of the Institute, the main research outcomes are reflected in the "Two Books One Journal". The so-called "Two Books" include Book Series of Materials of Sinographic Texts

* ZHANG Bowei 張伯偉 is professor of Classical Chinese Literature at School of Liberal Arts, Nanjing University. 
域外漢籍資料叢書書 and Book Series of the Study of Sinographic Text 域外漢 籍研究叢; the so-called “One Journal” is Journal of Studies on Sinographic Texts 域外漢籍研究集刊. Since 2005, Professor Zhang Bowei edits the world first academic journal focusing on the research of Sinographic Texts: "Journal of Studies on Sinographic Texts". This journal is published annually, published by Beijing Zhonghua Book Company, mainly focusing on academic research. It assembles academic papers and book reviews on language, literature, history, religion, ideology study in Chinese. Each volume of the "Collection" has 400,000-500,000 words in length, and the authors of the articles span the mainland China, Taiwan, Hong Kong and South Korea, Japan, Vietnam, Singapore, the United States, and other countries. So far, the "Journal" has been published in 15 volumes.

In 2007, Nanjing University Institute for the Study of Asian Classics in Chinese held "The First International Conference on Sinographic Texts Studies" in mainland China in East Asia. More than a hundred scholars from countries and regions including Korea, Japan, United States, mainland China, Hong Kong, and Taiwan attended this conference, and submitted approximately one hundred high-quality academic papers. This conference attracted widespread attention from scholars at home and abroad, and drew numerous responses from the field of Sinographic Texts studies. (For more information, please see "Notes of Attending Nanjing University International Conference on Sinographic Texts in East Asia" by Mizuguchi Motoki, and "International Conference on Sinographic Texts in East Asia" by Kono Kimiko [published on The Journal of Oriental Studies, vol.105, 2008].) In 2009, A Collection of Papers Presented at the First Nanjing University International Conference on Sinographic Texts in East Asia was published by Zhonghua Book Company. Ten years later, in the year of 2017 Nanjing University witnessed "The Senond International Conference on Sinographic Texts Studies" held in Nanjing, and more than 100 scholars from all over the world attend the conference, especially many young scholars participated this great enterprise. The collections of this Conference will be published by Zhonghua Book Company next year.

In 2005 and 2010, on periodicals Study and Exploration and Chinese Social Sciences Today, the Institute launched two discussions among scholars at home and abroad on Sinographic texts studies, including the intension and extension of the studies, the object of study, study method, research topics, and the significance of the studies. These discussions helped to deepen the understanding of Sinographic Texts studies.

For example, Liu Chunyin, the director of the library for the Institute of Chinese Literature and Philosophy, Academia Sinica, says: "In 2000, Nanjing University formally established the Institute for the Study of Asian Classics in 
Chinese, which is the first specialized research institution on Sinographic texts in the world. Till now, it has published the Journal of Studies on Sinographic Texts as well as the 'Book Series of the Study of Sinographic Text.' This institute promotes a systematic study on the dissemination, collation, cultural exchange of Sinographic texts, focusing on the research area on Sinographic texts by exploring the significances of the Sinographic texts in relation to the study of the entire Sinosphere." (Cited from "Editorial Principles for Summary: Using The Summary of Bibliography of Vietnam Hán Nôm Documents and its Addendum as An Example," Information Management for Buddhist Libraries, No.46, December 2007, p.72.)

Professor Shizunaga Takeshi from the Department of Literary Studies at Kyushu University in Japan also once states, "As for academic research, there is no 'national border!' However, in reality, as with scholars studying Japanese literature, Chinese scholars who study their own literature are also trapped in a blurry view that is sealed off by a diversity of invisible national borders. In such an academic context, we saw the rise of a new research method for sinology in mainland China - that is, the 'study of Sinographic texts' proposed by Professor Zhang Bowei, director of the Institute for the Study of Asian Classics in Chinese at Nanjing University. I personally think that this method is built upon the critique of the traditional research method that only focuses on one's own literature and domestic literary documents. It is a new academic paradigm that shows one's attempt to break the 'national border' of academic research. Professor Zhang Bowei's proposal of this concept, illustrates that his hope that China-based researchers of Chinese literature would be able to break the shackles of the 'national border,' expanding one's view of vision to Japan, Korea and other neighboring countries that have preserved texts written in Chinese language. Therefore, the 'study of Sinographic texts' is not a glorious buzzword, on the contrary, it is a methodology for all of us to understand the 'way of ruling' of China and the entire East Asian area. The so-called 'sinology,' therefore, should not be a field that only focuses on domestic China, but should also encompass more diversified perspectives and various ways of thinking that are border-crossing for the consideration of China in relation to the entire East Asian region-this should be the essence of "sinology'." (Cited from "A New Method in the Field of Chinese Studies," in Tōhō [Oriental], no.348, Feb 2010, published by Tōhō shoten in Japan.)

Professor Shizunaga Takeshi also points out, "In 2005, the Journal of Studies on Sinographic Texts edited by Professor Zhang Bowei in Nanjing University was officially founded. Subsequently, led by Professor Zhang Bowei, Dr. Jin Chengyu, Dr. Cai Yi and others have launched a book series entitled the 'Book Series on the Study of Sinographic Texts.' Therefore, with 
the publication of the journal and the book series every year, the Institute for the Study of Asian Classics in Chinese from Nanjing University has been continuously providing academic community with the latest research results in the field of the study on Sinographic texts." (Cited from "Sinographic Texts as an East Asian Commonality," Bungaku kenkyu [Studies in Literature], no. 107, October 2010, published by Kyushu University.)

Chen Qinghao, researcher of the CNRS also pointed out that:

In 2000, the Institute for the Study of Asian Classics in Chinese was founded in Nanjing University, which can be seen as the beginning of a new era for the study of Asian Classics in Chinese. In 2005, the institute launched the Journal of Studies on Sinographic Texts. From the same year, two sets of books, Book Series of Materials of Sinographic Texts and Book Series of the Study of Sinographic Text, started to be chiefly complied by the institute. Since then, a complete research system for the study of Sinographic Texts s in Chinese has been formed. Furthermore, in 2007, the International Symposium on the Study of Sinographic Texts in Chinese has been successfully held, followed by the publication of the conference papers. As the first specialized research institution in this field at home and abroad, the Institute, which bases its study on philology, pays attention to accumulating materials and cultivating research talents. With abundant materials, as well as collating information and research achievements published in the relevant bulletin and two series, the Institute has a promising future. (Thoughts at three decades of the overall study of the Chinese culture, Book Review by Zhonghua Book Company, 2011 (5) )

At present, adhering to the academic idea proposed by Prof. Zhang Bowei, which regards the circle of the Chinese culture as a method, the Institute deals with research problems in the realm of the Chinese culture. Hereupon, a brand-new literature foundation has been built. Taking extensive mastery and in-depth understanding of literature as the premise, the Institute put forward a number of issues with new academic significance. It will get rid of the chronic illness haunting the humanistic studies in East Asia over the past century which follows the Western academic ideas and methods to observe and study the Oriental learning. It will also provide the international academic community with academic ideas and methods different from the West. Therefore, it is reasonable to expect that such research ideas will be revolutionary and epoch-making for the East Asian literature and cultural studies. 